\title{
Polarity in Stem Cell Division: Asymmetric Stem Cell Division in Tissue Homeostasis
}

\author{
Yukiko M. Yamashita ${ }^{1,2}$, Hebao Yuan ${ }^{1}$, Jun Cheng ${ }^{3}$, and Alan J. Hunt ${ }^{3}$ \\ ${ }^{1}$ Life Sciences Institute, Center for Stem Cell Biology, University of Michigan, Ann Arbor, Michigan 48109 \\ ${ }^{2}$ Department of Cell and Developmental Biology, University of Michigan, Ann Arbor, Michigan 48109 \\ ${ }^{3}$ Department of Biomedical Engineering, Center for Ultrafast Optical Science, University of Michigan, \\ Ann Arbor, Michigan 48109 \\ Correspondence: yukikomy@umich.edu
}

\begin{abstract}
Many adult stem cells divide asymmetrically to balance self-renewal and differentiation, thereby maintaining tissue homeostasis. Asymmetric stem cell divisions depend on asymmetric cell architecture (i.e., cell polarity) within the cell and/or the cellular environment. In particular, as residents of the tissues they sustain, stem cells are inevitably placed in the context of the tissue architecture. Indeed, many stem cells are polarized within their microenvironment, or the stem cell niche, and their asymmetric division relies on their relationship with the microenvironment. Here, we review asymmetric stem cell divisions in the context of the stem cell niche with a focus on Drosophila germ line stem cells, where the nature of niche-dependent asymmetric stem cell division is well characterized.
\end{abstract}

\begin{abstract}
symmetric cell division allows stem cells to Aself-renew and produce another cell that undergoes differentiation, thus providing a simple method for tissue homeostasis. Stem cell self-renewal refers to the daughter(s) of stem cell division maintaining all stem cell characteristics, including proliferation capacity, maintenance of the undifferentiated state, and the capability to produce daughter cells that undergo differentiation. A failure to maintain the correct stem cell number has been speculated to lead to tumorigenesis/tissue hyperplasia via stem cell hyperproliferation or tissue degeneration/aging via a reduction in stem cell number or activity (Morrison and Kimble 2006; Rando 2006). This necessity changes
\end{abstract}

during development. The stem cell pool requires expansion earlier in development, whereas maintenance is needed later to sustain tissue homeostasis.

There are two major mechanisms to sustain a fixed number of adult stem cells: stem cell niche and asymmetric stem cell division, which are not mutually exclusive. Stem cell niche is a microenvironment in which stem cells reside, and provides essential signals required for stem cell identity (Fig. 1A). Physical limitation of niche "space" can therefore define stem cell number within a tissue. Within such a niche, many stem cells divide asymmetrically, giving rise to one stem cell and one differentiating cell, by placing

Editors: Rong Li and Bruce Bowerman

Additional Perspectives on Symmetry Breaking in Biology available at www.cshperspectives.org

Copyright (C) 2010 Cold Spring Harbor Laboratory Press; all rights reserved; doi: 10.1101/cshperspect.a001313

Cite this article as Cold Spring Harb Perspect Biol 2010;2:a001313 
Y.M. Yamashita et al.

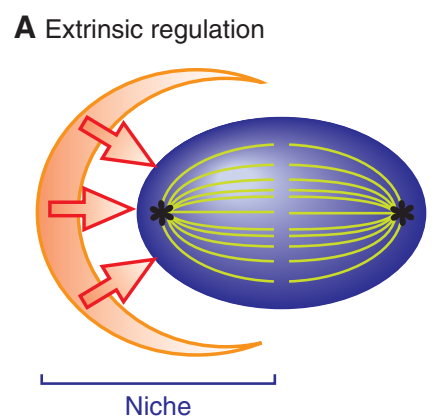

B Intrinsic regulation

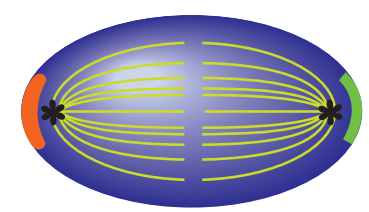

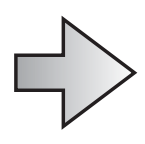

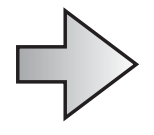

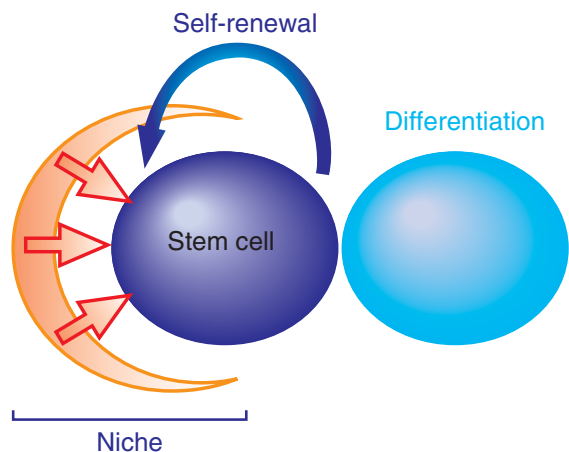

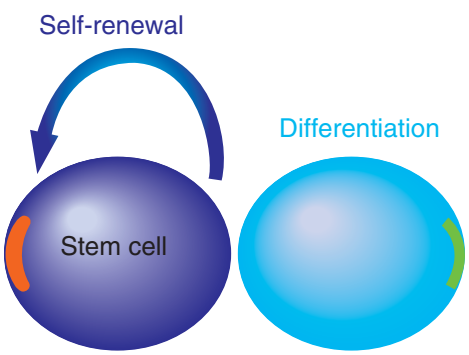

Figure 1. Mechanisms of asymmetric stem cell division. (A) Asymmetric stem cell division by extrinsic fate determinants (i.e., the stem cell niche). The two daughters of stem cell division will be placed in distinct cellular environments either inside or outside the stem cell niche, leading to asymmetric fate choice. $(B)$ Asymmetric stem cell division by intrinsic fate determinants. Fate determinants are polarized in the dividing stem cells, which are subsequently partitioned into two daughter cells unequally, thus making the division asymmetrical. Self-renewing (red line) and/or differentiation promoting (green line) factors may be involved.

one daughter inside and another outside of the niche, respectively (Fig. 1A). Nevertheless, some stem cells divide asymmetrically, apparently without the niche. For example, in Drosophila neuroblasts, cell-intrinsic fate determinants are polarized within a dividing cell, and subsequent partitioning of such fate determinants into daughter cells in an asymmetric manner results in asymmetric stem cell division (Fig. 1B) (see Fig. 3A and Prehoda 2009).

In this review, we focus primarily on asymmetric stem cell divisions in the Drosophila germ line as the most intensively studied examples of niche-dependent asymmetric stem cell division. We also discuss some examples of stem cell division outside Drosophila, where stem cells are known to divide asymmetrically or in a niche-dependent manner.

\section{DROSOPHILA MALE GERM LINE STEM CELLS}

\section{Signaling in the Male Germ Line Stem Cell (GSC) Niche}

The Drosophila male and female germ lines have served as ideal model systems for studying the regulation of stem cell behavior and asymmetric stem cell division controlled by the microenvironment or stem cell niche. Drosophila male germ line stem cells (GSCs) reside in a stem cell niche whose major components are hub cells and cyst stem cells (CySCs; historically called cyst progenitor cells) (Fig. 2A). Hub cells are generally thought to be postmitotic, attached to the apical wall of the testis, and consist of 8-16 somatically derived cells (Hardy et al. 1979). These hub cells are surrounded by 7-12 GSCs, such that each one is physically attached to the hub and encapsulated 

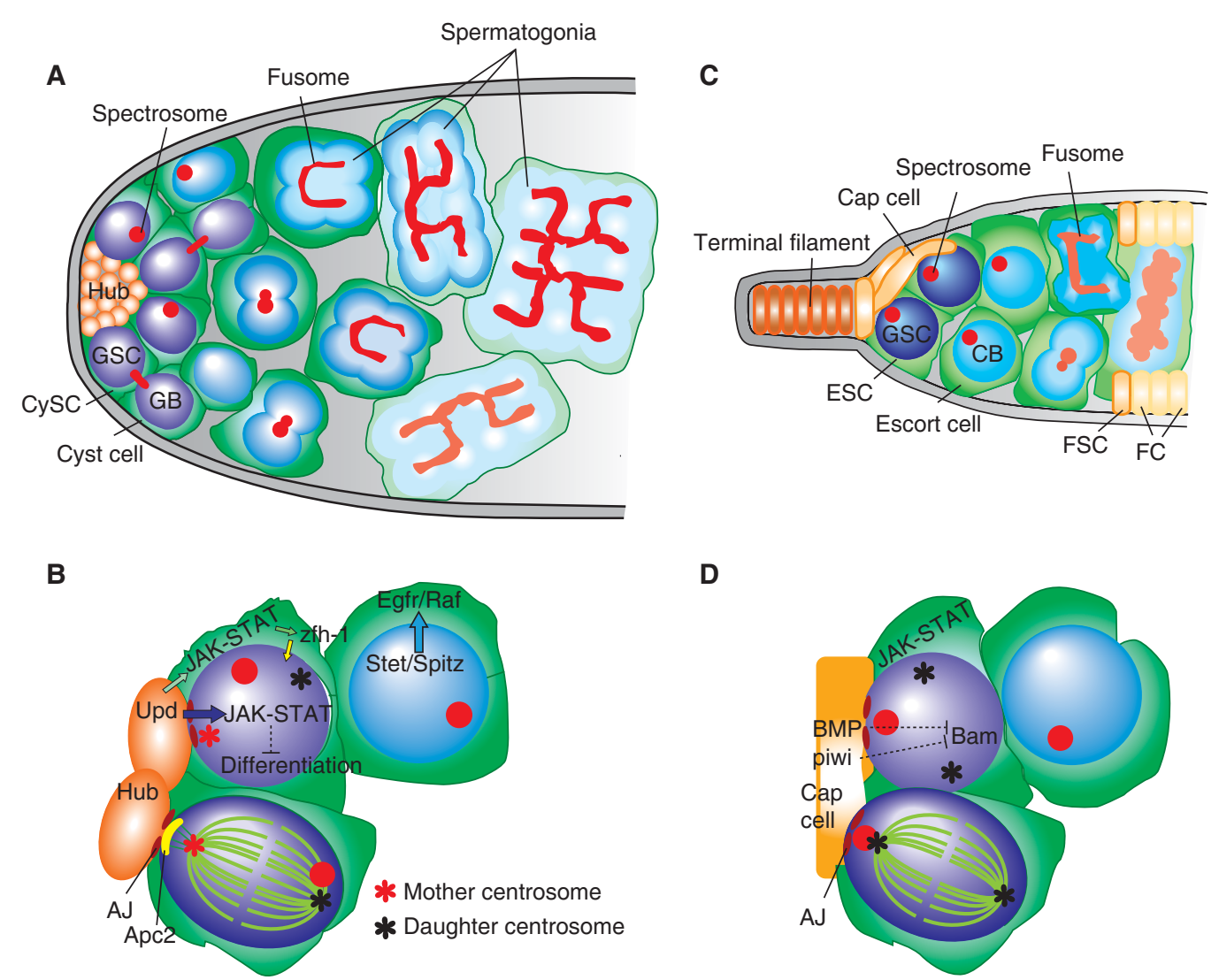

Figure 2. The anatomy of the Drosophila germ line stem cell (GSC) niche and asymmetric stem cell division. (A) Male GSC niche: GSCs and cyst stem cells (CySCs) are attached to hub cells via adherens junctions (AJs). GSCs divide asymmetrically to self-renew and produce a gonialblast (GB) that initiates the differentiation program. The GB further undergoes four synchronous, transit-amplifying divisions to yield 16 spermatogonia interconnected by the fusome. The spectrosome is a spherical version of the fusome observed in GSCs. A pair of CySCs encapsulates the GSCs and provides signals required for GSC identity. Similar to GSCs, CySCs divide asymmetrically to self-renew and produce cyst cells. Cyst cells exit the cell cycle, a pair of which encapsulates the GB and spermatogonia to promote differentiation. (B) Asymmetric stem cell division of male GSC by centrosome orientation: Upd ligand is secreted from hub cells to activate the JAK-STAT pathway in GSCs and CySCs to specify their stem cell identity. The Zfh-1 transcription factor controls CySC identity. Together with hub cells, CySCs dictate GSC identity. The mitotic spindle is oriented toward the hub cells via positioning of the centrosome. Spectrosomes in male GSCs are not oriented with respect to the hub cells during interphase. EGFR signaling ensures the encapsulation of germ cells by cyst cells. (C) Female GSC niche: GSCs are attached to the cap cells via adherens junctions. GSCs divide asymmetrically to self-renew and produce a cystoblast $(\mathrm{CB})$ that initiates differentiation. The $\mathrm{CB}$ divides four times to give rise to 16 germ cells interconnected by the fusome, only one of which becomes an oocyte, whereas the remaining 15 cells become nurse cells. Escort stem cells (ESCs) encapsulate the GSC, while their daughters, escort cells, encapsulate the developing germ cells. Escort cells are later replaced by follicle cells, which are daughters of follicle stem cells (FSCs). (D) Asymmetric stem cell division of female GSC by the spectrosome: BMP signaling and Piwi controls GSC identity via niche-GSC interaction. The mitotic spindle is oriented toward the cap cells via anchoring of one spindle pole to the spectrosome, which localizes consistently to the apical side of GSCs. (Figures are adapted and modified from Fuller and Spradling 2007.) 
Y.M. Yamashita et al.

by a pair of CySCs, which also maintain contact with the hub via cell processes. Male GSC division produces one GSC and one gonialblast, which then undergoes transit-amplifying divisions as spermatogonia (Fig. 2A). Spermatogonia become spermatocytes, which commit to meiosis and further differentiate into spermatids. Similar to GSCs, CySCs also divide asymmetrically, producing one CySC and one cyst cell. A pair of CySCs encapsulates a GSC, whereas a pair of cyst cells encapsulates developing gonialblast, spermatogonia, and spermatocytes. Cyst cells that encapsulate these developing germ cells do not divide, and differentiating germ cells undergo transitamplification as well as further development (i.e., meiosis and spermiogenesis) within the cyst encapsulated by a pair of cyst cells.

The interaction between germ cells and somatic cells is important for their normal functions. Self-renewal of GSCs and CySCs is specified by the JAK-STAT signaling pathway, which is activated by the ligand Unpaired (Upd) following secretion by hub cells (Fig. 2B) (Kiger et al. 2001; Tulina and Matunis 2001; Leatherman and Dinardo 2008). Consistently, overexpression of Upd in germ cells (GSCs and spermatogonia) or somatic cells (CySCs and cyst cells) hinders proper differentiation and results in tumor-like accumulation of undifferentiated, stem-like cells, whereas mutants of JAK or STAT lead to a rapid loss of GSCs and CySCs in a cellautonomous manner (Kiger et al. 2001; Tulina and Matunis 2001; Leatherman and Dinardo 2008). In addition, BMP signaling, which plays an important role in female GSC identity (see the following discussion), is implicated in stem cell self-renewal and/or proliferation of spermatogonia (Shivdasani and Ingham 2003; Kawase et al. 2004; Schulz et al. 2004), suggesting a conserved mechanism of stem cell selfrenewal/differentiation in both sexes. Because Upd acts within a short range (Harrison et al. 1998), only cells closely attached to the hub maintain their stem cell identity, and those displaced away from the hub initiate differentiation. Indeed, it has been demonstrated that cell adhesion between GSCs and hub cells, as well as between CySCs and hub cells, is required for stem cell maintenance, presumably by keeping cells together to take advantage of short-range self-renewal signals. The majority of adherens junctions in GSCs are clumped at the hub-GSC interface. These include the Drosophila epithelial cadherin (DE-cadherin) and the $\beta$-catenin homolog Armadillo (Yamashita et al. 2003). Consistently, loss-offunction mutant clones of shotgun, which encodes DE-cadherin, result in a rapid loss of GSCs and CySCs in a cell autonomous manner, suggesting a role for DE-cadherin in stem cell maintenance (Voog et al. 2008). As described in more detail in the following discussion, GSC mitotic spindle is oriented toward the hub-GSC interface, playing a key role in determining daughter cell positions with respect to the niche, and thus their fates.

In addition to regulatory signals from hub cells, the soma-germ communication between GSCs and CySCs (and/or spermatogonia and cyst cells) also plays an important role in specifying the fate of a germ cell (i.e., stem cell selfrenewal vs. commitment to differentiation) (Fig. 2B). Loss-of-function mutations in the epidermal growth factor receptor (EGFR) or its downstream mediator Raf in cyst cells leads to hyperproliferation of cells with GSC or gonialblast characteristics (Kiger et al. 2000; Tran et al. 2000). Furthermore, mutation in germ cells of either stet, a component of the EGFR signaling pathway, or spitz, an EGFR ligand, leads to a failure in the encapsulation of germ cells by cyst cells, causing defects in germ cell differentiation (Schulz et al. 2002; Sarkar et al. 2007). EGFR signaling appears to control germ cell-somatic cell (CySCs or cyst cells) attachment by modulating a balance between the Racl and Rhol small GTPases. Strikingly, Sakar et al. demonstrated that overexpression of a dominant negative form of Rho1 in somatic cells rescues the spitz mutant phenotype, allowing somatic cells to encapsulate germ cells successfully (Sarkar et al. 2007). This implies that EGFR signaling regulates germ cell identity primarily by controlling cell-cell interactions. Moreover, these results indicate that EGFR signaling does not provide 
the self-renewal signal per se, but allows other self-renewal signaling to be transduced by placing germ and soma in close contact.

It was recently discovered that CySCs play an instructive and dominant role in GSC specification. Although overexpression of an active form of JAK kinase in the germ line does not cause GSC or CySC tumors, such expression in cyst cells results in tumorigenesis (Leatherman and Dinardo 2008). Zfh-1, a transcription factor whose expression is restricted to CySCs and quickly down-regulated in cyst cells, was identified as a downstream target of the JAK-STAT pathway. Indeed, overexpression of $\mathrm{Zfh}-1$ in cyst cells leads to the hyperproliferation of CySCs, which in turn results in hyperproliferation of GSCs (Leatherman and Dinardo 2008). Taken together, these data demonstrate that CySCs play an instructive role in specifying GSC identity to the germ cells they encapsulate. These studies illuminate the complexity of cell-cell interactions in cell fate determination, in particular the interaction between two stem cell populations.

Asymmetric Stem Cell Division in the Niche: Spindle Orientation by the Centrosome

Theoretically, the limited availability in space provided by the short-range self-renewal signals that specify stem cell identity should be sufficient to sustain a fixed number of stem cells without depletion or hyperproliferation. However, GSCs appear to have adopted additional internal machinery to tightly regulate the balance between their self-renewal and differentiation.

The mitotic spindle is the macromolecular machinery involved in segregating chromosomes into two daughter cells. The spindle orientation determines the cleavage plane orientation (reviewed by Eggert et al. 2006), and thus delimits the spatial arrangement of the two daughter cells. The centrosome is an essential component of the cellular machinery that directs mitotic spindle formation and determines its orientation (reviewed by Kellogg et al. 1994). The asymmetric behavior of centrosomes plays a critical role in proper asymmetric stem cell division in Drosophila GSCs (Yamashita et al. 2003; Yamashita et al. 2007) and neuroblasts (Rebollo et al. 2007; Rusan and Peifer 2007).

In male Drosophila GSCs, the mitotic spindle is oriented perpendicular to the hub-GSC interface, leading to asymmetric division with the daughter cells inside and outside the niche (Fig. 2B) (Yamashita et al. 2003; Yamashita et al. 2007). Specifically, the mother centrosome is located near the apical cortical region adjacent to the hub cells. The newly duplicated daughter centrosome moves toward the distal end by the onset of mitosis. The mother centrosome may be captured and remains anchored by interphase astral microtubules via adherens junctions such as DE-cadherin and Armadillo formed at the hub-GSC interface. The unusually early centrosome separation in male GSCs, which occurs right after centrosome duplication, rather than at the G2/M transition, suggests that GSCs may be exploiting the difference in microtubule-anchoring activity between mother and daughter centrosomes before the daughter centrosome matures to anchor microtubules. Consistent with the idea that the mother centrosome is anchored to the hub-GSC interface by microtubules, loss-of-function mutants of Centrosomin (Cnn) exhibit dysfunctional centrosome orientation and randomization of the mother-daughter choice. Cnn is an integral component of the pericentriolar material (PCM) and is required for anchoring many centrosomal components onto centrosomes (Megraw et al. 1999; Vaizel-Ohayon and Schejter 1999; Megraw et al. 2001; Megraw et al. 2002). Indeed, electron microscopy has revealed that the mother centrosome tends to have more interphase astral microtubules, some of which span from the PCM area to adherens junction (Yamashita et al. 2007). In addition, homologs of adenomatous polyposis coli (Apc1 or Apc2) are required for centrosome orientation in GSCs (Yamashita et al. 2003). Apc proteins associate with microtubule plus ends and the actin cytoskeleton, and play important roles in cellular adhesion (McCartney et al. 1999; Yu et al. 1999; McCartney et al. 2001; 
Y.M. Yamashita et al.

Akong et al. 2002a; Akong et al. 2002b; Hamada and Bienz 2002). In GSCs, Apcl colocalizes with spindle poles and Apc2 localizes to the hub-GSC interface, the latter of which may bind both microtubules and the adherens junction component $\beta$-catenin, thus linking the centrosome and adherens junction (Yamashita et al. 2003). It is currently unknown if these Apc genes are required for GSC self-renewal or not.

It should be noted that the asymmetry of GSC division solely relies on the asymmetric microenvironment (i.e., inside vs. outside of the niche-signaling range), because germ line cells that initiated differentiation (spermatogonia in testis and cystocytes in ovary) can revert or "dedifferentiate" into GSCs once they are brought back to the stem cell niche (Brawley and Matunis 2004; Kai and Spradling 2004). Once established during development, this asymmetric microenvironment sustains tissue homeostasis.

How such tissue architecture is established during development is a fascinating but less understood process. In Drosophila male germ line, this process appears to occur during embryonic development when primordial germ cells (PGCs, precursors of GSCs) and the somatic gonadal precursors (SGPs, precursors of hub, CySC, and cyst cells) coalesce (DeFalco et al. 2003; Wawersik et al. 2005). PGCs that happen to be juxtaposed to the SGP/hub cells acquire GSC identity, whereas other PGCs directly undergo differentiation. Concomitantly, cell-cell contacts ( presumably adherens junctions as in adult flies) are formed between GSCs and the SGP/hub cells, and as soon as GSC fates are established, their divisions are oriented and asymmetric (Tanentzapf et al. 2007). The establishment of GSC asymmetry by interacting with the niche component cells occurs in female germ line as well (Asaoka and Lin 2004).

\section{Asymmetric Stem Cell Division and Aging}

Stem cell maintenance is important for tissue homeostasis. A decline in either stem cell number or proliferation may lead to tissue degeneration associated with disease or aging.
Indeed, a decline in stem cell number attributable to changes in both the stem cells themselves and the stem cell niche has been reported in male and female germ line stem cells (Boyle et al. 2007; Pan et al. 2007). In addition to these, we have demonstrated that changes in stem cell orientation precede a decline in stem cell number in the male germ line, leading to tissue aging. As Drosophila males age, GSCs with misoriented centrosomes accumulate progressively ( $\sim 40 \%$ of total GSCs at day 30 ), when a decrease in stem cell number is still subtle. Misoriented GSCs do not undergo mitosis until the centrosome orientation is corrected (Cheng et al. 2008). This combination of a slowdown in cell division (i.e., G2/M transition) in misoriented GSCs with an increase in misoriented GSCs is speculated to cause a decline in spermatogenesis with age. The fact that GSCs resume cell cycle progression on correction of the centrosome orientation implies that there might be a novel checkpoint mechanism to monitor centrosome orientation and ensure an asymmetric outcome of stem cell division.

Remarkably, some misoriented GSCs originate from dedifferentiation (Cheng et al. 2008). Throughout Drosophila adulthood, individual GSCs are lost at a certain rate (Xie and Spradling 1998; Wallenfang et al. 2006). Dedifferentiation of partially differentiated germ cells was proposed as a mechanism to replenish the stem cell pool (Brawley and Matunis 2004; Kai and Spradling 2004). Such dedifferentiated GSCs were found to have a high incidence of centrosome misorientation (Cheng et al. 2008). Therefore, whereas dedifferentiation may play a key role in maintaining GSC number with age, reduced mitotic activity because of misorientation results in less GSC division and thus reduced production of differentiating germ cells (i.e., spermatogonia). These results suggest that a decline in stem cell activity comes from an imperfect yet compensating activity of the tissue to replenish the stem cell pool (Spradling 2008). It should be noted that when germ cells commit to differentiation, they lose the mother centrosome that stays in the GSCs. Thus, dedifferentiated GSCs do not possess the very old mother centrosomes, even 
though they may have a mother centrosome that has undergone multiple cell cycles since they committed to differentiation. Thus, one can speculate that the high frequency of centrosome misorientation in dedifferentiated GSCs may reflect the fact that they do not have the "very old" centrosomes or the "centrosomal Eve" that was generated early during embryogenesis (Yamashita and Fuller 2008). Additional research is required to elucidate whether any physical differences between the "centrosomal Eve" in native GSCs and the "not-so-old mother centrosome" in dedifferentiated GSCs exist.

\section{DROSOPHILA FEMALE GERM LINE STEM CELLS}

\section{Signaling in the Female GSC Niche}

The niche of female GSCs shares numerous similarities with male GSCs in both structure and signaling. Female GSCs and their niche reside in a structure called the germarium, located at the anterior tip of each ovariole, the individual egg-producing unit of the ovary (also see Roth and Lynch 2009). This niche is composed of three kinds of somatic cell populations: terminal filament (TF) cells at the tip of the germarium, cap cells at the base of the terminal filament cells, and escort stem cells (ESCs) (Fig. 2C) (Decotto and Spradling 2005). A few ovarian GSCs, typically two to three, are tightly associated with five to seven cap cells via adherens junctions and receive short-range signals secreted from the niche cells (i.e., cap cells and TF) for self-renewal. Loss of adherens junctions between cap cells and GSCs causes the stem cells to migrate away from the cap cells and undergo differentiation (Song and Xie 2002). Between four to six ESCs are also anchored to the cap cells and contact GSCs. ESCs are believed to be the female counterpart of CySCs in males, both of which encapsulate GSCs and contribute to their fate via JAK-STAT signaling (Decotto and Spradling 2005; Leatherman and Dinardo 2008). Similar to CySCs in males, ESCs also produce daughter cells called escort cells, which encapsulate the differentiating germ line cysts until they undergo apoptosis and are replaced by follicle cells.

The BMP signaling pathway is the best understood mechanism shown to be both necessary and sufficient for the maintenance of female GSCs. Two BMP-like ligands, dpp and gbb, are secreted from cap cells, which then activate the signaling pathway in GSCs and prevent them from differentiating by repressing the transcription of Bam, a key differentiationpromoting factor (Fig. 2D) (Chen and McKearin 2003a; Chen and McKearin 2003b; Song et al. 2004; Chen and McKearin 2005). Loss of GSCs is observed in dpp mutants because of premature differentiation and slower division rates. Dpp overexpression completely blocks differentiation of cystoblasts, resulting in GSC-like tumors (Xie and Spradling 1998). Transient induction of Bam expression by heatshock, either in the second instar larval ovariole, where dpp is activated in the entire germ cell compartment, or in an adult ovariole, where dpp is ectopically expressed in somatic cells, induces differentiation of GSCs (Ohlstein and McKearin 1997). More interestingly, the induced cystocytes gradually undergo dedifferentiation over time and are converted into functional germ line stem cells in the presence of $\mathrm{dpp}$ in the surrounding somatic cell population, demonstrating the dominance of the niche in maintaining GSCs (Kai and Spradling 2004). Fs(1)Yb (Yb)-Piwimediated signaling is also required for GSC maintenance and was initially thought to be BMP-independent because dpp overexpression did not rescue Piwi mutant defects or show similar effects as $\mathrm{Yb}$ or Piwi overexpression. $\mathrm{Yb}$ exerts its function by regulating Piwi expression in TF cells and cap cells (King and Lin 1999; King et al. 2001). Piwi is involved in germ line development, stem cell identity, epigenetic regulation, and transposon silencing in many systems. Piwi binds to a complex class of small noncoding RNAs called Piwi-interacting RNAs (piRNAs) to execute its functions (Lin 2007; Ghildiyal and Zamore 2009; Lin and Yin 2009). In Yb-null mutant females, GSCs lose their ability to self-renew by either differentiating into germ line cysts without any division or 
Y.M. Yamashita et al.

undergoing a limited number of abnormal cell divisions. Piwi mutants phenocopy $\mathrm{Yb}$ mutants. Piwi and $\mathrm{Yb}$ overexpression increases GSC-like or cystoblast-like germ cells (King and Lin 1999; King et al. 2001). Surprisingly, two recent reports demonstrate that BMP- and Piwi-mediated signaling converge to act synergistically to silence Bam (Chen and McKearin 2005; Szakmary et al. 2005). Interestingly, the JAK-STAT pathway is also found to be essential in ESCs to maintain female GSC identity (Decotto and Spradling 2005). How these different signaling pathways regulate GSC selfrenewal cooperatively remains to be addressed.

Asymmetric Stem Cell Division in the Niche: Spindle Orientation by the Spectrosome

Similar to male GSCs, female GSCs undergo asymmetric cell division to maintain the balance between self-renewal and differentiation. Normally, a GSC divides asymmetrically to produce one daughter that stays associated with the cap cells to retain stem cell identity and another that moves away from the cap cells to become a cystoblast, which commits to differentiation. The cystoblast undergoes four additional rounds of mitoses with incomplete cytokinesis to form an interconnected 16-cell cyst. In contrast to male GSCs, whose spindle orientation is set up by centrosome positioning during interphase, the spindle of a female GSC is oriented through anchorage of one spindle pole to the spectrosome, a germ cell specific subcellular organelle, which is always located on the apical side of the GSC (Fig. 2D) (Deng and Lin 1997; also see Roth and Lynch 2009). Eliminating the spectrosome randomizes spindle orientation in the dividing female GSC (Deng and Lin 1997). Cytoplasmic dynein has been shown to be involved in coupling the mitotic spindle to the membrane skeletal proteins in the spectrosome/fusome (McGrail and Hays 1997). In agreement with the distinct mechanisms for orienting the mitotic spindle in male versus female GSCs (the centrosome vs. spectrosome, respectively), spectrosome positioning in male GSCs is random during interphase (Yamashita et al. 2003), and centrosome positioning is reported to be random during interphase in female GSCs (Stevens et al. 2007). Also, whereas female GSCs have been observed to undergo symmetric stem cell division on elimination of neighboring GSCs (Xie and Spradling 2000), male GSCs rarely undergo these events (Cheng et al. 2008), further suggesting distinct mechanisms for stem cell orientation and asymmetric stem cell division.

The molecular identity of the spectrosome/ fusome has yet to be fully resolved. Nevertheless, this structure is always referred to as a membranous compartment associated with membrane skeletal proteins and microtubules with vesicle trafficking function similar to the ER and/or Golgi (Deng and Lin 1997; Roper and Brown 2004; Snapp et al. 2004; Roper 2007; Lighthouse et al. 2008). In addition, cyclin A has been reported to localize to the spectrosome/fusome, controlling the number of germ cell divisions before entering mitosis and implying a cell cycle role for the fusome (Lilly et al. 2000).

\section{OTHER DROSOPHILA STEM CELLS AND STEM CELL POLARITY}

Until recently, the Drosophila adult body, except for the gonad, was regarded as mostly postmitotic without much necessity for replenishment or adult stem cells. However, recent studies revealed many somatic stem cells in Drosophila adult tissues, including midgut (Micchelli and Perrimon 2006; Ohlstein and Spradling 2006; Ohlstein and Spradling 2007; Lin et al. 2008), hindgut (Takashima et al. 2008), and malpighian tubule stem cells (Singh et al. 2007). Midgut stem cells divide with an oriented spindle (relatively fixed orientation with respect to basement membrane) (Ohlstein and Spradling 2007); however, whether this spindle orientation is used as a means for asymmetric division or for any other aspects of stem cell biology remains to be determined. Underlying muscle cells that associate with midgut stem cells control stem cell identity via wingless signaling (Lin et al. 2008), and midgut stem cells can respond to injury to repair the damaged tissue quickly (Amcheslavsky et al. 2009). 
This illuminates a striking parallel between Drosophila and mammalian intestinal stem cells (Casali and Batlle 2009).

Recent characterization of ovarian follicle stem cells revealed a novel type of stem cell microenvironment (Nystul and Spradling 2007). Follicle stem cells (FSCs) produce follicle cells that encapsulate developing oocyte and nurse cells, taking the place of escort cells after they undergo apoptosis. Each ovariole appears to contain exactly two FSCs residing in separate niches on opposite sides of the germarium (Fig. 2C). The most striking feature of FSCs is the apparent lack of fixed niche components. Instead, the location of FSCs appears to be determined relative to the location of other cells. Further investigation is required to determine if FSCs maintain a polarity toward any of their interacting cells. Although several signaling pathways, including the Hedgehog, Wingless, and BMP pathways, as well as adherens junctions, have been implicated in FSC maintenance (Forbes et al. 1996; Song and Xie 2002; Song and Xie 2003; Kirilly et al. 2005), it is still unknown how these signals crosstalk or which cells provide polarity cues to FSCs. In spite of the progress made thus far in understanding Drosophila adult stem cells, the polarity and asymmetric division of stem cells remain to be elucidated.

\section{VERTEBRATE STEM CELLS: HOW MUCH CAN WE APPLY LESSONS FROM DROSOPHILA?}

Although many stem cell populations have been identified and the number continues to grow, only a few of them have been characterized in terms of their polarity/asymmetry and relationship with their niche. In many cases, the presence and/or nature of the stem cell niche itself is still ambiguous. Below, we focus on some stem cell populations outside Drosophila, where the cell polarity and/or mitotic spindle orientation is known.

\section{Mammalian (mouse) Neuroepithelial Cells}

All neurons and macroglial cells of the mammalian central nervous system are derived from neuroepithelial (NE) cells, which function as stem cells. During development, NE cells shift from proliferative, symmetric divisions to neurogenic, asymmetric divisions. This asymmetry derives from the inheritance of a tiny apical surface area (apical cortex), which contains Cadherin (i.e., the cell that inherited this apical cortex retains stem cell potential). Because this apical cortex is very small, spindle orientation does not need to be strictly parallel or vertical with respect to the apical surface to determine a symmetric versus asymmetric outcome, respectively (Fig. 3B) (Kosodo et al. 2004). Indeed, NE cells frequently divide asymmetrically with an almost parallel spindle with only a slight tilt, unequally segregating the apical cortex into two daughters. This is in clear contrast to the Drosophila neuroblast, where the drastic spindle orientation (horizontal vs. vertical) is a determinant for asymmetric stem cell division (Fig. 3A) (for more details, see $\mathrm{Yu}$ et al. 2006 and Chia et al. 2008). In the normal Drosophila neuroblasts, the mitotic spindle is formed perpendicularly with respect to the apical and basal "crescents" that contain fate determinants and complexes that regulate spindle orientation, leading to asymmetric division (Fig. 3A). In contrast, in the neuroectoderm cells, from which neuroblasts derive, and in the mutant neuroblasts defective in spindle orientation, the mitotic spindle is formed in parallel to these crescents, resulting in symmetric divisions.

These observations in the Drosophila highlight the correlation between spindle orientation and cell fates, and raise the question as to how mammalian NE cells divide symmetrically versus asymmetrically in spite of their narrow range of spindle orientations. Yet, a shift from symmetric (proliferative) to asymmetric (neurogenic) divisions occurs during development, clearly implying that there must exist a mechanism that determines symmetric versus asymmetric division. Interestingly, disruption of the function of LGN, a homolog of Pins required for spindle orientation in the Drosophila neuroblasts, leads to dramatic randomization of spindle orientation with a wider range of spindle orientation than normally 
Y.M. Yamashita et al.
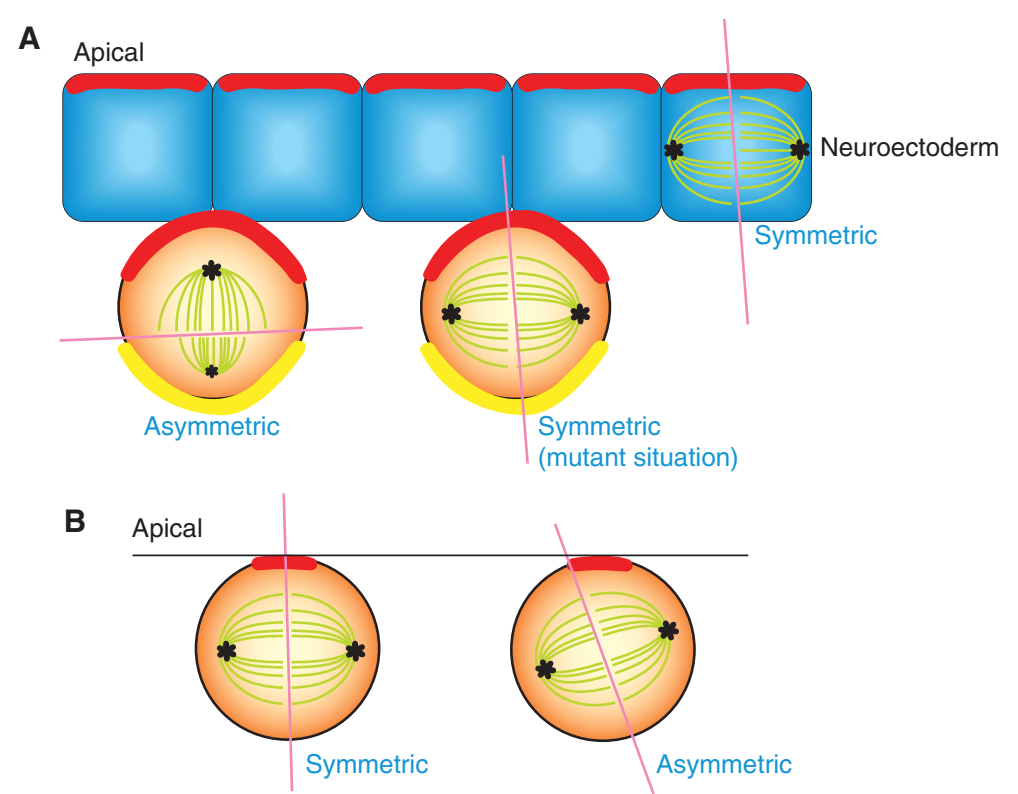

Figure 3. Spindle orientation and asymmetric division in Drosophila and mouse neuronal stem cells. $(A)$ In Drosophila neuroblast, spindle is oriented perpendicular to the apical crescent (red line) that contains the Baz (Par3)-Par6-aPKC complex, as well as Pins, Insc, and Gai, leading to asymmetric stem cell division. The apical crescent is required for spindle orientation, the basal crescent formation and spindle size asymmetry (i.e., the apical half is larger than the basal half). The basal crescent (yellow line) contains fate determinants that promote/allow differentiation such as Numb, Miranda, and Prospero. In the mutants that are defective in spindle orientation, the apical and basal crescents are bisected into two daughters, leading to symmetric stem cell division. Neuroectoderm cells (from which neuroblasts are derived) also have the apical complex, except for Insc. They divide symmetrically by orienting mitotic spindle parallel to the apical crescent. Ectopic expression of Insc in these cells result in the recruitment of Pins to the apical cortex, leading to perpendicular spindle orientataion (Yu et al. 2000). (B) In mammalian (mouse) neuroepithelial cells, the mode of cell division shifts from symmetric to asymmetric during development. The stem cell identity is determined by inheritance of a tiny apical cortex containing cadherin (red line), and thus mitotic spindle does not have to tilt significantly to divide asymmetrically.

observed in mammalian NE cells (Konno et al. 2008). This raises the intriguing possibility that a "parallel" spindle is the result of an elaborate regulation of spindle orientation, and that a slight tilt in orientation within a small range is actively controlled to dictate symmetric versus asymmetric NE divisions.

Interestingly, recent studies found another asymmetry during $\mathrm{NE}$ cell divisions: Prominin-1, or CD133, localizes to the midbody ring of dividing NE cells with an interesting asymmetry, correlated with the mode of stem cell division. Prominin-1 is expressed in many adult stem cells and cancer stem cells, although its function remains unclear. Before the neurogenic stage of development, when NEcells are proliferating by symmetric divisions, the Prominin-1-containing midbody ring is excluded from both daughters, being released into the extracellular space (Dubreuil et al. 2007; Farkas and Huttner 2008). Once neurogenesis begins later in development, NE cells start dividing asymmetrically and the Prominin-1-containing ring is inherited by the stem cell (NE cell), whereas the daughter fated to differentiate is devoid of this ring. This strong correlation implies that the Prominin-1-containing midbody ring might be somehow involved in stem cell behavior such as stem cell potential or asymmetric stem 
cell division. Although intriguing, further work is required to examine this hypothesis.

\section{Mammalian Skin Stem Cells}

Skin stem cells reside within the basal layer of the epidermis and contact the basement membrane. These cells tend to divide symmetrically with parallel spindles relative to the basement membrane when the tissue (i.e., skin surface area) is increasing in size during development. Then, they undergo asymmetric division by shifting the spindle orientation from parallel to perpendicular once stratification starts later in development and during tissue homeostasis (Lechler and Fuchs 2005). The basement membrane provides key features such as integrinmediated focal adhesion and growth factors. Thus, asymmetric inheritance of contact with the basement membrane automatically dictates asymmetric stem cell division and stratification of epidermal cells. It was further shown that these epidermal cells control spindle orientation in a similar manner to Drosophila neuroblasts. LGN, mInsc, the mammalian homolog of Drosophila Insc, and Par3 are localized asymmetrically in the dividing epidermal stem cells (Lechler and Fuchs 2005).

\section{Conclusion: Parallels between Mammalian and Drosophila Stem Cells}

The two examples above provide a very nice parallel between the mammalian and Drosophila stem cell systems. First, spindle orientation is the main theme for determining symmetric or asymmetric stem cell division regardless of whether it is controlled by intrinsic or extrinsic cues. The mechanism of how spindle orientation is determined varies from one system to another. These two mammalian examples discussed above resemble Drosophila neuroblasts in that these stem cells appear to use spindle rotation, which presumably allows for greater flexibility in the decision about symmetric versus asymmetric stem cell division. However, such flexibility may leave room for stem cells to divide symmetrically without regulation, leading to tumorigenesis. It should be noted that all of mammalian examples represent stem cells in a developing tissue at the transition from symmetric to asymmetric stem cell division. Therefore, it would be interesting to investigate how stem cell division is controlled in mammals once they are established as adult stem cells, where stereotyped asymmetric stem cell division would be favored. Drosophila GSC studies have focused on the adult stage, and thus may represent a more stereotyped mechanism to ensure asymmetric stem cell divisions.

Asymmetric stem cell division is fundamental to tissue homeostasis, and requires elaborate mechanisms to establish and maintain cell asymmetry in the context of the resident tissue. Recent discoveries from Drosophila and mammalian tissues have enriched our knowledge about how stem cells divide asymmetrically. In general, stem cells reside in their special microenvironment (or niche) that specifies stem cell identity. Resident stem cells are often closely associated with the niche component via cell-cell contact, such as adherens junctions. In such a context of tissue architecture and cell-cell signaling, stem cells are polarized such that they orient mitotic spindles to divide symmetrically or asymmetrically. Although our knowledge on stem cell division is far from comprehensive, the parallels between Drosophila and mammalian systems are striking, suggesting that asymmetric stem cell division is an evolutionarily conserved process. Further studies investigating the parallels between these model organisms and humans will lead to a better understanding of the mechanism of stem cell division, and of diseases that are caused by dysfunctional stem cell division.

\section{ACKNOWLEDGMENTS}

We thank the members of the Yamashita laboratory for insightful discussions. This work was supported by a University of Michigan start-up fund, the March of Dimes Basil O'Conner Starter Scholar Research Award, the Searle Scholar Program, and National Institutes of Health (NIH) R01GM086481-01 (to Y.M.Y.), and NIH R01GM072006 (to A.J.H.). 
Y.M. Yamashita et al.

\section{REFERENCES}

Akong K, McCartney B, Peifer M. 2002b. Drosophila APC2 and $\mathrm{APC} 1$ have overlapping roles in the larval brain despite their distinct intracellular localizations. Dev Biol 250: 71 .

Akong K, Grevengoed E, Price M, McCartney B, Hayden M, DeNofrio J, Peifer M. 2002a. Drosophila APC2 and APC1 play overlapping roles in wingless signaling in the embryo and imaginal discs. Dev Biol 250: 91.

Amcheslavsky A, Jiang J, Ip YT. 2009. Tissue damage-induced intestinal stem cell division in Drosophila. Cell Stem Cell 4: 49-61.

Asaoka M, Lin H. 2004. Germline stem cells in the Drosophila ovary descend from pole cells in the anterior region of the embryonic gonad. Development 131: 5079-5089.

Boyle M, Wong C, Rocha M, Jones DL. 2007. Decline in selfrenewal factors contributes to aging of the stem cell niche in the Drosophila testis. Cell Stem Cell 1: 470-478.

Brawley C, Matunis E. 2004. Regeneration of male germline stem cells by spermatogonial dedifferentiation in vivo. Science 304: 1331-1334.

Casali A, Batlle E. 2009. Intestinal stem cells in mammals and Drosophila. Cell Stem Cell 4: 124-127.

Chen D, McKearin D. 2003a. Dpp signaling silences bam transcription directly to establish asymmetric divisions of germline stem cells. Curr Biol 13: 1786-1791.

Chen D, McKearin D. 2005. Gene circuitry controlling a stem cell niche. Curr Biol 15: 179-184.

Chen D, McKearin DM. 2003b. A discrete transcriptional silencer in the bam gene determines asymmetric division of the Drosophila germline stem cell. Development 130: 1159-1170.

Cheng J, Turkel N, Hemati N, Fuller MT, Hunt AJ, Yamashita YM. 2008. Centrosome misorientation reduces stem cell division during ageing. Nature 456: 599-604.

Chia W, Somers WG, Wang H. 2008. Drosophila neuroblast asymmetric divisions: Cell cycle regulators, asymmetric protein localization, and tumorigenesis. J Cell Biol 180: 267-272.

Decotto E, Spradling AC. 2005. The Drosophila ovarian and testis stem cell niches: Similar somatic stem cells and signals. Dev Cell 9: 501-510.

DeFalco TJ, Verney G, Jenkins AB, McCaffery JM, Russell S, Van Doren M. 2003. Sex-specific apoptosis regulates sexual dimorphism in the Drosophila embryonic gonad. Dev Cell 5: 205-216.

Deng W, Lin H. 1997. Spectrosomes and fusomes anchor mitotic spindles during asymmetric germ cell divisions and facilitate the formation of a polarized microtubule array for oocyte specification in Drosophila. Dev Biol 189: 79-94.

Dubreuil V, Marzesco AM, Corbeil D, Huttner WB, Wilsch-Brauninger M. 2007. Midbody and primary cilium of neural progenitors release extracellular membrane particles enriched in the stem cell marker prominin-1. J Cell Biol 176: 483-495.

Eggert US, Mitchison TJ, Field CM. 2006. Animal cytokinesis: From parts list to mechanisms. Annu Rev Biochem 75: $543-566$.
Farkas LM, Huttner WB. 2008. The cell biology of neural stem and progenitor cells and its significance for their proliferation versus differentiation during mammalian brain development. Curr Opin Cell Biol 20: 707-715.

Forbes AJ, Lin H, Ingham PW, Spradling AC. 1996. Hedgehog is required for the proliferation and specification of ovarian somatic cells prior to egg chamber formation in Drosophila. Development 122: 1125-1135.

Fuller MT, Spradling AC. 2007. Male and female Drosophila germline stem cells: Two versions of immortality. Science 316: 402-404.

Ghildiyal M, Zamore PD. 2009. Small silencing RNAs: An expanding universe. Nat Rev Genet 10: 94-108.

Hamada F, Bienz M. 2002. A Drosophila APC tumour suppressor homologue functions in cellular adhesion. Nat Cell Biol 4: 208-213.

Hardy RW, Tokuyasu KT, Lindsley DL, Garavito M. 1979. The germinal proliferation center in the testis of Drosophila melanogaster. J Ultrastruct Res 69: 180-190.

Harrison DA, McCoon PE, Binari R, Gilman M, Perrimon N. 1998. Drosophila unpaired encodes a secreted protein that activates the JAK signaling pathway. Genes Dev 12: 3252-3263.

Kai T, Spradling A. 2004. Differentiating germ cells can revert into functional stem cells in Drosophila melanogaster ovaries. Nature 428: 564-569.

Kawase E, Wong MD, Ding BC, Xie T. 2004. Gbb/Bmp signaling is essential for maintaining germline stem cells and for repressing bam transcription in the Drosophila testis. Development 131: 1365-1375.

Kellogg DR, Moritz M, Alberts BM. 1994. The centrosome and cellular organization. Annu Rev Biochem 63: 639-674.

Kiger AA, White-Cooper H, Fuller MT. 2000. Somatic support cells restrict germline stem cell self-renewal and promote differentiation. Nature 407: 750-754.

Kiger AA, Jones DL, Schulz C, Rogers MB, Fuller MT. 2001. Stem cell self-renewal specified by JAK-STAT activation in response to a support cell cue. Science 294: 2542-2545.

King FJ, Lin H. 1999. Somatic signaling mediated by fs(1) Yb is essential for germline stem cell maintenance during Drosophila oogenesis. Development 126: 1833-1844.

King FJ, Szakmary A, Cox DN, Lin H. 2001. Yb modulates the divisions of both germline and somatic stem cells through piwi- and hh-mediated mechanisms in the Drosophila ovary. Mol Cell 7: 497-508.

Kirilly D, Spana EP, Perrimon N, Padgett RW, Xie T. 2005. BMP signaling is required for controlling somatic stem cell self-renewal in the Drosophila ovary. Dev Cell 9: 651-662.

Konno D, Shioi G, Shitamukai A, Mori A, Kiyonari H, Miyata T, Matsuzaki F. 2008. Neuroepithelial progenitors undergo LGN-dependent planar divisions to maintain self-renewability during mammalian neurogenesis. Nat Cell Biol 10: 93-101.

Kosodo Y, Roper K, Haubensak W, Marzesco AM, Corbeil D, Huttner WB. 2004. Asymmetric distribution of the apical plasma membrane during neurogenic divisions of mammalian neuroepithelial cells. Embo J 23: 2314-2324.

Leatherman JL, Dinardo S. 2008. Zfh-1 controls somatic stem cell self-renewal in the Drosophila testis and 
nonautonomously influences germline stem cell selfrenewal. Cell Stem Cell 3: 44-54.

Lechler T, Fuchs E. 2005. Asymmetric cell divisions promote stratification and differentiation of mammalian skin. Nature 437: 275-280.

Lighthouse DV, Buszczak M, Spradling AC. 2008. New components of the Drosophila fusome suggest it plays novel roles in signaling and transport. Dev Biol 317: 59-71.

Lilly MA, de Cuevas M, Spradling AC. 2000. Cyclin A associates with the fusome during germline cyst formation in the Drosophila ovary. Dev Biol 218: 53-63.

Lin H. 2007. piRNAs in the germ line. Science 316: 397.

Lin H, Yin H. 2009. A novel epigenetic mechanism in Drosophila somatic cells mediated by PIWI and piRNAs. Cold Spring Harb Symp Quant Biol 73: 273-281.

Lin G, Xu N, Xi R. 2008. Paracrine Wingless signalling controls self-renewal of Drosophila intestinal stem cells. Nature 455: 1119-1123.

McCartney BM, Dierick HA, Kirkpatrick C, Moline MM, Baas A, Peifer M, Bejsovec A. 1999. Drosophila APC2 is a cytoskeletally-associated protein that regulates wingless signaling in the embryonic epidermis. J Cell Biol 146: 1303-1318.

McCartney BM, McEwen DG, Grevengoed E, Maddox P, Bejsovec A, Peifer M. 2001. Drosophila APC2 and Armadillo participate in tethering mitotic spindles to cortical actin. Nat Cell Biol 3: 933-938.

McGrail M, Hays TS. 1997. The microtubule motor cytoplasmic dynein is required for spindle orientation during germline cell divisions and oocyte differentiation in Drosophila. Development 124: 2409-2419.

Megraw TL, Kao LR, Kaufman TC. 2001. Zygotic development without functional mitotic centrosomes. Curr Biol 11: 116-120.

Megraw TL, Kilaru S, Turner FR, Kaufman TC. 2002. The centrosome is a dynamic structure that ejects PCM flares. J Cell Sci 115: 4707-4718.

Megraw TL, Li K, Kao LR, Kaufman TC. 1999. The centrosomin protein is required for centrosome assembly and function during cleavage in Drosophila. Development 126: $2829-2839$.

Micchelli CA, Perrimon N. 2006. Evidence that stem cells reside in the adult Drosophila midgut epithelium. Nature 439: 475-479.

Morrison SJ, Kimble J. 2006. Asymmetric and symmetric stem-cell divisions in development and cancer. Nature 441: 1068-1074.

Nystul T, Spradling A. 2007. An Epithelial Nihce in the Drosophila ovary undergoes long-range stem cell replacement. Cell Stem Cell 1: 277-285.

Ohlstein B, McKearin D. 1997. Ectopic expression of the Drosophila Bam protein eliminates oogenic germline stem cells. Development 124: 3651-3662.

Ohlstein B, Spradling A. 2006. The adult Drosophila posterior midgut is maintained by pluripotent stem cells. Nature 439: 470-474.

Ohlstein B, Spradling A. 2007. Multipotent Drosophila intestinal stem cells specify daughter cell fates by differential notch signaling. Science 315: 988-992.
Pan L, Chen S, Weng C, Call G, Zhu D, Tang H, Zhang N, Xie T. 2007. Stem cell aging is controlled both intrinsically and extrinsically in the Drosophila ovary. Cell Stem Cell 1: 458-469.

Prehoda KE. 2009. Polarization of Drosophila neuroblasts during asymmetric division. Cold Spring Harb Perspect Biol 1: a001388.

Rando TA. 2006. Stem cells, ageing and the quest for immortality. Nature 441: 1080-1086.

Rebollo E, Sampaio P, Januschke J, Llamazares S, Varmark H, Gonzalez C. 2007. Functionally unequal centrosomes drive spindle orientation in asymmetrically dividing Drosophila neural stem cells. Dev Cell 12: 467-474.

Roper K. 2007. Rtnll is enriched in a specialized germline ER that associates with ribonucleoprotein granule components. J Cell Sci 120: 1081-1092.

Roper K, Brown NH. 2004. A spectraplakin is enriched on the fusome and organizes microtubules during oocyte specification in Drosophila. Curr Biol 14: 99-110.

Roth S, Lynch JA. 2009. Symmetry breaking during Drosophila oogenesis. Cold Spring Harb Perspect Biol 1: a001891.

Rusan NM, Peifer M. 2007. A role for a novel centrosome cycle in asymmetric cell division. J Cell Biol 177: $13-20$.

Sarkar A, Parikh N, Hearn SA, Fuller MT, Tazuke SI, Schulz C. 2007. Antagonistic roles of Rac and Rho in organizing the germ cell microenvironment. Curr Biol 17: $1253-1258$.

Schulz C, Kiger AA, Tazuke SI, Yamashita YM, Pantalena-Filho LC, Jones DL, Wood CG, Fuller MT. 2004. A Misexpression Screen Reveals Effects of bag-ofmarbles and TGFbeta Class Signaling on the Drosophila Male Germ-Line Stem Cell Lineage. Genetics 167: 707-723.

Schulz C, Wood CG, Jones DL, Tazuke SI, Fuller MT. 2002. Signaling from germ cells mediated by the rhomboid homolog stet organizes encapsulation by somatic support cells. Development 129: 4523-4534.

Shivdasani AA, Ingham PW. 2003. Regulation of stem cell maintenance and transit amplifying cell proliferation by tgf-Beta signaling in Drosophila spermatogenesis. Curr Biol 13: 2065-2072.

Singh SR, Liu W, Hou SX. 2007. The adult Drosophila malpighian tubules are maintained by multipotent stem cells. Cell Stem Cell 1: 191-203.

Snapp EL, Iida T, Frescas D, Lippincott-Schwartz J, Lilly MA. 2004. The fusome mediates intercellular endoplasmic reticulum connectivity in Drosophila ovarian cysts. Mol Biol Cell 15: 4512-4521.

Song X, Xie T. 2002. DE-cadherin-mediated cell adhesion is essential for maintaining somatic stem cells in the Drosophila ovary. Proc Natl Acad Sci 99: 14813-14818.

Song X, Xie T. 2003. Wingless signaling regulates the maintenance of ovarian somatic stem cells in Drosophila. Development 130: 3259-3268.

Song X, Wong MD, Kawase E, Xi R, Ding BC, McCarthy JJ, Xie T. 2004. Bmp signals from niche cells directly repress transcription of a differentiation-promoting gene, bag of 
Y.M. Yamashita et al.

marbles, in germline stem cells in the Drosophila ovary. Development 131: 1353-1364.

Spradling AC. 2008. Stem cells: Makeshift sperm production. Nature 456: 583-585.

Stevens NR, Raposo AA, Basto R, St Johnston D, Raff JW. 2007. From stem cell to embryo without centrioles. Curr Biol 17: 1498-1503.

Szakmary A, Cox DN, Wang Z, Lin H. 2005. Regulatory Relationship among piwi, pumilio, and bag-of-marbles in Drosophila Germline Stem Cell Self-Renewal and Differentiation. Curr Biol 15: 171-178.

Takashima S, Mkrtchyan M, Younossi-Hartenstein A, Merriam JR, Hartenstein V. 2008. The behaviour of Drosophila adult hindgut stem cells is controlled by Wnt and Hh signalling. Nature 454: 651-655.

Tanentzapf G, Devenport D, Godt D, Brown NH. 2007. Integrin-dependent anchoring of a stem-cell niche. Nat Cell Biol 9: 1413-1418.

Tran J, Brenner TJ, DiNardo S. 2000. Somatic control over the germline stem cell lineage during Drosophila spermatogenesis. Nature 407: 754-757.

Tulina N, Matunis E. 2001. Control of stem cell self-renewal in Drosophila spermatogenesis by JAK-STAT signaling. Science 294: 2546-2549.

Vaizel-Ohayon D, Schejter ED. 1999. Mutations in centrosomin reveal requirements for centrosomal function during early Drosophila embryogenesis. Curr Biol 9: $889-898$

Voog J, D’Alterio C, Jones DL. 2008. Multipotent somatic stem cells contribute to the stem cell niche in the Drosophila testis. Nature 454: 1132-1136.
Wallenfang MR, Nayak R, DiNardo S. 2006. Dynamics of the male germline stem cell population during aging of Drosophila melanogaster. Aging Cell 5: 297-304.

Wawersik M, Milutinovich A, Casper AL, Matunis E, Williams B, Van Doren M. 2005. Somatic control of germline sexual development is mediated by the JAK/ STAT pathway. Nature 436: 563-567.

Xie T, Spradling AC. 1998. Decapentaplegic is essential for the maintenance and division of germline stem cells in the Drosophila ovary. Cell 94: 251-260.

Xie T, Spradling AC. 2000. A niche maintaining germ line stem cells in the Drosophila ovary. Science 290: 328-330.

Yamashita YM, Fuller MT. 2008. Asymmetric centrosome behavior and the mechanisms of stem cell division. $J$ Cell Biol 180: 261-266.

Yamashita YM, Jones DL, Fuller MT. 2003. Orientation of asymmetric stem cell division by the APC tumor suppressor and centrosome. Science 301: 1547-1550.

Yamashita YM, Mahowald AP, Perlin JR, Fuller MT. 2007. Asymmetric inheritance of mother versus daughter centrosome in stem cell division. Science 315: 518-521.

Yu F, Kuo CT, Jan YN. 2006. Drosophila neuroblast asymmetric cell division: Recent advances and implications for stem cell biology. Neuron 51: 13-20.

Yu X, Waltzer L, Bienz M. 1999. A new Drosophila APC homologue associated with adhesive zones of epithelial cells. Nat Cell Biol 1: 144-151.

Yu F, Morin X, Cai Y, Yang X, Chia W. 2000. Analysis of partner of inscuteable, a novel player of Drosophila asymmetric divisions, reveals two distinct steps in inscuteable apical localization. Cell 100: 399-409. 


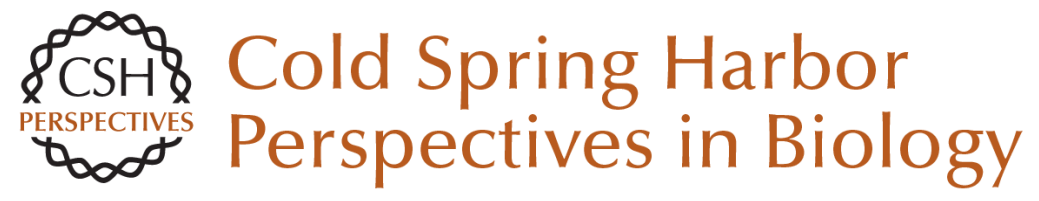

\section{Polarity in Stem Cell Division: Asymmetric Stem Cell Division in Tissue Homeostasis}

Yukiko M. Yamashita, Hebao Yuan, Jun Cheng and Alan J. Hunt

Cold Spring Harb Perspect Biol 2010; doi: 10.1101/cshperspect.a001313 originally published online August 5, 2009

Subject Collection Symmetry Breaking in Biology

Cytoskeletal Mechanisms for Breaking Cellular

Symmetry

R. Dyche Mullins

Symmetry Breaking in Biology

Rong Li and Bruce Bowerman

Planar Cell Polarity Signaling: The Developing Cell's Compass

Eszter K. Vladar, Dragana Antic and Jeffrey D. Axelrod

Cellular Polarity in Prokaryotic Organisms Jonathan Dworkin

Symmetry Breaking in Plants: Molecular Mechanisms Regulating Asymmetric Cell Divisions in Arabidopsis Jalean J. Petricka, Jaimie M. Van Norman and Philip N. Benfey

The Signaling Mechanisms Underlying Cell Polarity and Chemotaxis Fei Wang

Polarization of Drosophila Neuroblasts During Asymmetric Division Kenneth E. Prehoda
Polarity in Stem Cell Division: Asymmetric Stem Cell Division in Tissue Homeostasis

Yukiko M. Yamashita, Hebao Yuan, Jun Cheng, et al.

Symmetry Breaking in the Life Cycle of the Budding Yeast Brian D. Slaughter, Sarah E. Smith and Rong Li

Neuronal Polarity Sabina Tahirovic and Frank Bradke

\author{
Membrane Organization and Dynamics in Cell \\ Polarity \\ Kelly Orlando and Wei Guo \\ Cellular Symmetry Breaking during \\ Caenorhabditis elegans Development \\ Edwin Munro and Bruce Bowerman
}
Symmetry Breaking During Drosophila Oogenesis Siegfried Roth and Jeremy A. Lynch

Widely Conserved Signaling Pathways in the Establishment of Cell Polarity Luke Martin McCaffrey and lan G. Macara

For additional articles in this collection, see http://cshperspectives.cshlp.org/cgi/collection/

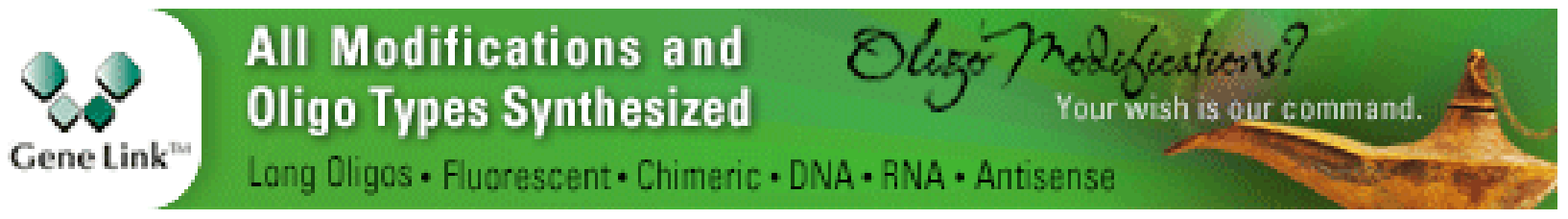



Physical Model of Cellular Symmetry Breaking
Jasper van der Gucht and Cécile Sykes
Shaping Fission Yeast with Microtubules Fred Chang and Sophie G. Martin

For additional articles in this collection, see http://cshperspectives.cshlp.org/cgi/collection/

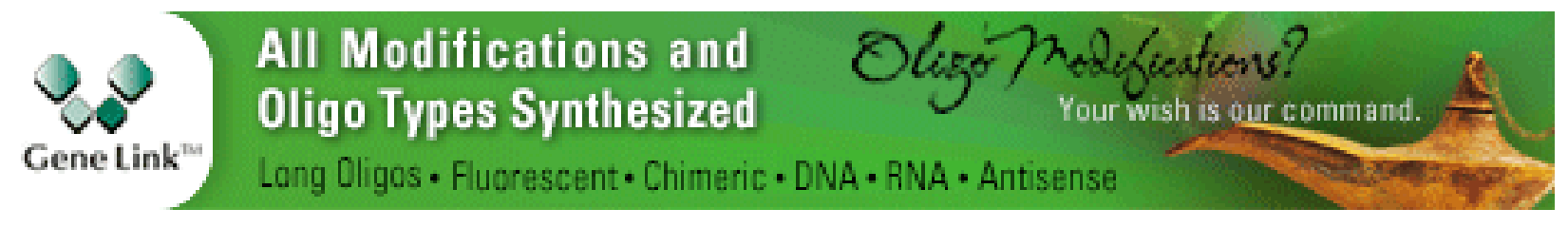

Copyright @ 2010 Cold Spring Harbor Laboratory Press; all rights reserved 